\title{
EMT-related gene expression is positively correlated with immunity and may be derived from stromal cells in osteosarcoma
}

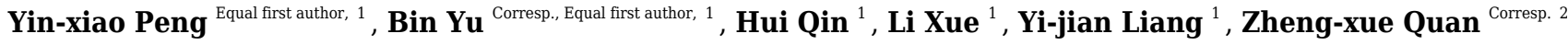 \\ 1 Department of orthopedics, The third people's hospital of Chengdu, Chengdu, Sichuan, China \\ 2 Department of orthopedics, The First Affiliated Hospital of Chongqing Medical University, Chongqing, Chongqing, China \\ Corresponding Authors: Bin Yu, Zheng-xue Quan \\ Email address: 768832674@qq.com, quanzx18@126.com
}

Background: Osteosarcoma is the most common type of bone cancer in children and young adults. Recent studies have shown a correlation between epithelial-mesenchymal transition (EMT)-related gene expression and immunity in human cancers. Here, we investigated the relationship among EMT, immune activity, stromal activity and tumor purity in osteosarcoma. Methods: We defined EMT gene signatures and evaluated immune activity and stromal activity based on the gene expression and clinical data from three independent microarray datasets. These factors were evaluated by single sample Gene Set Enrichment Analyses (sSGSEA) and the ESTIMATE tool. Finally, we analyzed the key source of EMT gene expression in osteosarcoma using microarray datasets from the GEO and human samples that we collected. Results: EMT-related gene expression was positively correlated with immune and stromal activity in osteosarcoma. Tumor purity was negatively correlated with EMT, immune activity and stromal cells. We further demonstrated that high EMT gene expression could significantly predict poor overall survival (OS) and recurrence-free survival (RFS) in osteosarcoma patients, while high immune activity cannot. However, combining these factors could have further prognostic value for osteosarcoma patients in terms of OS and RFS. Finally, we found that stromal cells may serve as a key source of EMT gene expression in osteosarcoma. Conclusion: The results of this study reveal that the expression of EMT genes and immunity are positively correlated, but these signatures convey disparate prognostic information. Furthermore, the results indicate that EMT-related gene expression may be derived from stromal rather than epithelial cancer cells. 
1 EMT-related gene expression is positively correlated with immunity and may be derived

2 from stromal cells in osteosarcoma

3

4 Running title: EMT and Osteosarcoma

5

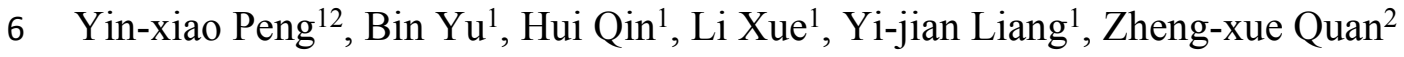

7 'Department of orthopedics, The third people's hospital of Chengdu, Chengdu, Sichuan, China

$8{ }^{2}$ Department of orthopedics, The First Affiliated Hospital of Chongqing Medical University,

9 Chongqing, China

10

11 Corresponding Authors:

12 Zheng-xue Quan ${ }^{2}$

13 No.1 Yixueyuan road, Yuzhong District, Chongqing, 400016, China

14 E-mail: quanzx18@126.com

15 Bin $\mathrm{Yu}^{1}$

16 No. 82 Qinglong Street, Chengdu, Sichuan, 601131, China

17 E-mail: 768832674@qq.com

18

19

Yin-xiao Peng and Bin Yu contributed equally to this work

20 
21

22

\section{Abstract}

Background: Osteosarcoma is the most common type of bone cancer in children and young adults. Recent studies have shown a correlation between epithelial-mesenchymal transition (EMT)related gene expression and immunity in human cancers. Here, we investigated the relationship among EMT, immune activity, stromal activity and tumor purity in osteosarcoma.

Methods: We defined EMT gene signatures and evaluated immune activity and stromal activity based on the gene expression and clinical data from three independent microarray datasets. These factors were evaluated by single sample Gene Set Enrichment Analyses (ssGSEA) and the ESTIMATE tool. Finally, we analyzed the key source of EMT gene expression in osteosarcoma using microarray datasets from the GEO and human samples that we collected.

Results: EMT-related gene expression was positively correlated with immune and stromal activity in osteosarcoma. Tumor purity was negatively correlated with EMT, immune activity and stromal cells. We further demonstrated that high EMT gene expression could significantly predict poor overall survival (OS) and recurrence-free survival (RFS) in osteosarcoma patients, while high immune activity cannot. However, combining these factors could have further prognostic value for osteosarcoma patients in terms of OS and RFS. Finally, we found that stromal cells may serve as a key source of EMT gene expression in osteosarcoma.

Conclusion: The results of this study reveal that the expression of EMT genes and immunity are positively correlated, but these signatures convey disparate prognostic information. Furthermore, the results indicate that EMT-related gene expression may be derived from stromal rather than epithelial cancer cells.

Keywords: Osteosarcoma; EMT; Immunity; Stroma; Tumor purity 


\section{INTRODUCTION}

Osteosarcoma is the most common type of bone cancer in children and young adults and is responsible for approximately $9 \%$ of cancer deaths in children and adolescents aged 10-24 years(Biazzo \& De Paolis 2016; Maximov et al. 2019). Chemotherapy and surgical intervention can increase the 5-year survival rate to $60 \%-70 \%$. However, the 5-year survival rate is below $30 \%$ for patients who have metastatic disease at diagnosis or recurrence(Sayles et al. 2019; Shen et al. 2017). Thus, it is important to explore the mechanisms underlying osteosarcoma metastasis to improve patient prognosis.

Tumor metastasis begins with epithelial-mesenchymal transition (EMT), in which epithelial cells acquire a mesenchymal phenotype and become migratory and invasive(Shen et al. 2017; Wang et al. 2018). Loss of the epithelial marker E-cadherin and an increase in the expression of mesenchymal markers are characteristics of EMT. EMT promotes primary epithelial-like tumor cells to acquire invasive mesenchymal phenotypes during metastatic progression. This progression, along with increased motility and invasiveness, triggers the dissemination of metastatic cells from the tumor, which then infiltrate into the tumor vasculature(Lv et al. 2016). Hence, EMT-related genes are potential markers and therapeutic targets for osteosarcoma treatment.

A recent study showed that EMT is associated with immunity in human cancers(Gugnoni et al. 2017; Mitschke et al. 2019; Yeung \& Yang 2017). Increased expression of EMT markers in breast tumors is associated with increased immune infiltration into the tumor microenvironment(Kotiyal \& Bhattacharya 2014; Yeung \& Yang 2017). The presence of these immune cells then promotes immune evasion by the tumor cells, which is associated with tumor progression and metastasis(Singh \& Chakrabarti 2019). EMT status was found to be strongly associated with an inflammatory tumor microenvironment in non-small-cell lung cancer(Lou et al. 2016). Pancancer analysis revealed a strong correlation between EMT and immune activation(Mak et al. 2016). However, to the best of our knowledge, the relationship between EMT and the immune microenvironment in osteosarcoma has not been reported. What is the cellular origin of EMT- 
related gene signatures in osteosarcoma expression data? How do EMT genes and immune activity collectively impact survival in osteosarcoma patients?

In this study, we explored the relationship among EMT, immune activity, stromal activity and tumor purity in osteosarcoma based on gene expression and clinical data from three independent microarray datasets. These factors were evaluated by single-sample Gene Set Enrichment Analysis (ssGSEA) and the ESTIMATE tool. Finally, RT-qPCR was used to verify the results.

\section{MATERIALS AND METHODS}

Raw data

Three independent microarray datasets, namely, GSE36001, GSE39055 and GSE16091, were obtained from the GEO database (Gene Expression Omnibus, https://www.ncbi.nlm.nih.gov/geo/)(Kelly et al. 2013; Kresse et al. 2012; Paoloni et al. 2009). For GSE36001, we obtained 19 osteosarcoma cell lines and 6 normal samples, including two normal osteoblast samples and four normal bone samples. For GSE39055 and GSE16091, we obtained 37 and 34 human osteosarcoma samples, respectively.

\section{EMT score}

The EMT-related gene signature comprised 200 genes obtained from the gene set "HALLMARK_EPITHELIAL_MESENCHYMAL_TRANSITION" in The Molecular Signatures Database hallmark gene sets (MSigDB, software.broadinstitute.org/gsea/msigdb, supplementary material Table 2)(Subramanian et al. 2005). For each sample in TCGA, the EMT score was calculated by ssGSEA in R software 3.5.0 based on EMT-related gene expression(Hanzelmann et al. 2013). ssGSEA calculates the EMT gene set enrichment score per osteosarcoma sample as the normalized difference in empirical cumulative distribution functions of EMT-related gene expression ranks inside and outside the EMT gene set.

\section{Immune score, stromal score and tumor purity}

To explore the relationship among EMT, immune activity, stromal activity and tumor purity, 
100

101

102

103

104

105

106

107

108

109

110

111

112

113

114

115

116

117

118

119

120

121

122

123

124

125

126

we used the ESTIMATE package of R software 3.5.0 to evaluate the index(Yoshihara et al. 2013). The ESTIMATE package defines immune and stromal gene signatures to infer the proportion of the immune and stromal components from gene expression data and combines these individual components to estimate tumor purity. The stromal gene signature comprises 141 genes.

\section{Correlation analysis}

Correlation analysis was used for comparisons of two gene sets using GraphPad Prism 7. The Pearson $\mathrm{R}$ value and two-tailed $\mathrm{P}$ value are used to evaluate the correlation level. Compute the $\mathrm{R}$ value for $\mathrm{X}$ versus every $\mathrm{Y}$ data set.

\section{Expression analysis of EMT and stromal cell gene signature}

GSE36001 was used in the expression analysis of EMT-related and stromal cell gene signatures. The expression value was vst-transformed and quantile normalized. Fold change (FC) in EMT-related and stromal cell genes between tumor and normal samples was calculated. Student's t test was used for comparisons between two groups using GraphPad Prism 7.

\section{Survival analysis}

The overall survival (OS) and recurrence-free survival (RFS) data from GSE39055 was used for survival analysis. Kaplan-Meier survival curves and the log-rank test were used to assess the OS and RFS and index score or individual gene expression. The degree of OS and RFS were summarized by hazard ratios (HRs) and $P$ values, which were determined using GraphPad Prism 7. The method of determining cut-off values for grouping high and low levels was described in previous studies(Uhlen et al. 2017).

\section{RT-qPCR}

A total of 5 osteosarcoma tissues and their pair-matched adjacent stromal tissues were obtained with informed consent from patients who underwent radical resections at the Department of Orthopedics, The Third People's Hospital of Chengdu, China. Osteosarcoma and stromal tissues were derived from surgical resection of osteosarcoma. The expression levels of 25 EMT-related genes in the osteosarcoma and stromal samples were examined using real-time quantitative polymerase chain reaction (RT-qPCR). RNA was converted into cDNA using the PrimeScript ${ }^{\circledR}$ 
127

128

129

130

131

132

133

134

135

136

137

138

139

140

141

142

143

144

145

1st Strand cDNA Synthesis Kit (TaKaRa, Japan). Quantification of the cDNA template was performed with RT-qPCR using SYBR green as a fluorophore. The primer sequences of the 25 EMT-related genes examined in this study can be found in the supplementary material Table 1. All experimental procedures were approved by the Medical Ethics Committee of the Third People's Hospital of Chengdu.

\section{RESULTS}

\section{EMT-related genes, immune activity and stromal genes in osteosarcoma}

To determine the EMT score in osteosarcoma patients, we collected EMT-related genes from the gene set "Hallmark_epithelial_mesenchymal_transition" in MSigDB. The EMT score was calculated by ssGSEA according to gene expression in two independent microarray datasets (GSE39055 and GSE16091). Obviously, the expression level of EMT-related genes increased as the EMT score increased in GSE39055 and GSE16091 (Fig. 1A and B). To identify immune and stromal activity in osteosarcoma patients, the immune score and stromal score were calculated by ESTIMATE according to gene expression in GSE39055 and GSE16091. We found that the expression of immune-related genes and stromal related genes increased as the immune score (Fig. $1 \mathrm{C}$ and D) and stromal score (Fig. 1E and F) increased, respectively. These results suggest that the EMT, immune and stromal scores can be used to evaluate the expression of genes related to EMT, immune activity and stromal activity, respectively.

\section{EMT-related gene expression is positively correlated with immune and stromal activity in}

\section{osteosarcoma}

We explored the relationship among EMT, immune activity and stromal activity. The EMT score was positively correlated with the immune score in GSE39055 ( $\mathrm{R}=0.4433, P=0.0060)$ and GSE16091 ( $\mathrm{R}=0.38, P=0.0266$ ) (Fig. 2A and B). The EMT score was also positively correlated with the stromal scores in GSE39055 ( $\mathrm{R}=0.647, P<0.0001)$ and GSE16091 $(\mathrm{R}=0.6026, P=0.0002)$ (Fig. 2C and D). Next, we analyzed the relationship among tumor purity, EMT, immune activity and stromal activity. We found that the EMT score was negatively correlated with tumor purity in 
154

155

156

157

158

159

160

161

162

163

164

165

166

167

168

169

170

171

172

173

174

175

176

177

178

179

180

GSE39055 (R=-0.6125, $P<0.0001)$ and GSE16091 ( $\mathrm{R}=-0.5048, P=0.0023)$ (Fig. 2E and F). The immune score was negatively correlated with tumor purity in GSE39055 ( $\mathrm{R}=-0.9296, P<0.0001)$ and GSE16091 ( $\mathrm{R}=-0.932, P<0.0001)$ (Fig. 2G and H). The stromal score was also negatively correlated with tumor purity in GSE39055 $(\mathrm{R}=-0.8983, P<0.0001)$ and GSE16091 $(\mathrm{R}=-0.8974$, $P<0.0001$ ) (Fig. 2I and J). These results indicate that EMT-related gene expression in osteosarcoma may result from stromal cells in the tumor microenvironment rather than epithelial cancer cells.

\section{Expression of EMT-related genes and immune activity result in disparate clinical outcomes} in osteosarcoma patients

To explore the clinical value of EMT and immune activity in osteosarcoma, we analyzed OS and RFS in GSE39055. OS is an important endpoint, with the advantage of minimal ambiguity in defining an OS event(Liu et al. 2018), while RFS can represent disease recurrence under the influence of related factors. Fig. 3A-D demonstrates the association of OS with EMT and immune activity in osteosarcoma patients. A high EMT activity was associated with significantly poorer OS in osteosarcoma patients $(\mathrm{HR}=4.977, P=0.0025$, Fig. 3A). However, a high immune score was associated with improved OS in osteosarcoma patients, but this association was not significant $(\mathrm{HR}=0.4047, P=0.1726$, Fig. 3B). Although the EMT score was positively correlated with immune activity (Fig. 2A and B), disparate clinical outcomes were reported. Thus, we explored whether combining these factors would have further prognostic value in these patients. We found that a large difference between immune activity and the EMT score was associated with significantly improved $\mathrm{OS}$ in osteosarcoma patients $(\mathrm{HR}=0, P=0.029$, Fig. 3C). Fig. 3D shows the OS of four groups stratified according to both EMT and immune scores $(P=0.007)$. High immune activity and a low EMT score were associated with the best OS in osteosarcoma patients. However, low immune activity and a high EMT score were associated with significantly poorer OS in osteosarcoma patients.

Fig. 3E-G presents the association of RFS with EMT and immune activity in osteosarcoma. Similar to the results of OS in patients, a high EMT score was associated with significantly poorer 
181

182

183

184

185

186

187

RFS in osteosarcoma patients $(\mathrm{HR}=4.308, P=0.0098$, Fig. 3E). A high immune score was associated with better RFS in osteosarcoma patients, but this association was not significant (HR $=0.4816, P=0.1335$, Fig. 3F). A large difference between the immune activity and EMT scores was associated with significantly improved RFS in osteosarcoma patients $(\mathrm{HR}=0.2781, P=$ 0.0085, Fig. 3G). High immune activity and low EMT were associated with the best RFS in osteosarcoma patients, while low immune activity and high EMT were associated with the worst RFS (Fig. 3H).

\section{Stromal cells are a key source of EMT-related gene expression in osteosarcoma patients}

To explore the relationship between EMT-related and stromal cell gene signatures in osteosarcoma, we analyzed the expression of and clinical outcomes associated with each gene signature. First, we analyzed related gene expression in the GSE36001 dataset. At the individual gene level, the expression of EMT-related genes was higher than that of stromal cell genes; the mean value of EMT-related genes was 9.12, while that of stromal cell genes was $7.421(P<0.0001$, Fig. 4A-B). The FC in EMT-related genes was also higher than that in stromal cell genes; the mean value of EMT-related genes was -0.98 , while that of stromal cell genes was $-0.7(P<0.0001$, Fig. 4C-D). Next, we evaluated the prognostic significance of individual genes from EMT and stromal signatures. Ten of the top 15 genes and 11 of the top 15 genes most significantly associated with OS and RFS in GSE39055 were from the EMT-related gene set (Fig. 4E-F).

To verify our findings, we used RT-qPCR to detect the relative expression of the above 25 genes, including 18 EMT signature genes, 3 stromal signature genes and 4 genes belonging to both EMT-related and stromal signatures. We found that the expression of EMT-related genes was higher than that of stromal cell genes in human osteosarcoma samples (Fig. 4G), while the expression of stromal cell genes was higher than that of EMT-related genes in human stromal samples (Fig. 4H). Moreover, the FC in EMT-related genes was also higher than that in stromal cell genes (Fig. 4I). Therefore, although EMT and stromal activity were highly correlated in osteosarcoma (Fig. 2C-D), EMT-related genes were more strongly associated with survival than stromal cell genes. These results suggest that stromal cells may serve as a key source of EMT- 
208

209

210

211

212

213

214

215

216

217

218

219

220

221

222

223

224

225

226

227

228

229

230

231

232

233

234

related gene expression in osteosarcoma.

\section{DISCUSSION}

Osteosarcoma is the most common type of bone cancer in young people. The 5-year survival rate for patients who have metastatic disease is low(Chang 2019; Maximov et al. 2019; Shen et al. 2017). Tumor metastasis begins with EMT, which is a critical event in osteosarcoma metastasis(Jiang et al. 2019; Shen et al. 2017; Wang et al. 2018). Moreover, accumulating evidence has shown that EMT is associated with immunity in human cancers(Lou et al. 2016; Mak et al. 2016; Singh \& Chakrabarti 2019). However, to the best of our knowledge, the relationship between EMT and the immune microenvironment in osteosarcoma has not been reported. Here, we have shown that EMT was positively correlated with immune activity and that these parameters may hold prognostic value for osteosarcoma patients.

We defined EMT-related gene signatures and evaluated immune activity and stromal activity based on gene expression in two independent microarray datasets. EMT and immune activity demonstrated a positive correlation. A positive correlation was also observed between EMT and stromal cell activity. Furthermore, we found that tumor purity was negatively correlated with EMT, immune activity and stromal cells. Altogether, these results suggest that the expression of EMT-related genes in osteosarcoma may originate from stromal cells in the tumor microenvironment rather than from epithelial cancer cells. These findings are consistent with the findings from recent studies in urothelial cancer and colorectal cancer(Isella et al. 2015; Isella et al. 2016; Wang et al. 2018). Using the Cancer Genome Atlas (TCGA) urothelial cancer dataset, Wang et al. showed that gene expression-based measures of infiltrating T-cell abundance and EMT are positively correlated. Stromal cells are a major source of EMT-related gene expression in bulk urothelial cancer transcriptomes(Wang et al. 2018). Using colorectal cancer expression data from patient-derived xenografts, Isella C. et al. showed that upregulated EMT-related genes are also prominently expressed by stromal cells, suggesting that EMT-related genes could originate from stromal rather than epithelial cancer cells(Isella et al. 2015). 
We demonstrated that the expression of EMT-related genes and immune activity result in

236

237

238

239

240

241

242

243

244

245

246

247

248

249

250

251

252

253

254

255

256

257

258

259

260

261

262

disparate clinical outcomes in osteosarcoma patients. A high EMT score was associated with significantly poor OS and RFS in osteosarcoma patients, while a high immune score was associated with better OS and RFS in osteosarcoma patients, but the latter association was not significant. However, combining these factors had further prognostic value for osteosarcoma patients in terms of OS and RFS. We found that high immune activity and a low EMT score were associated with the best OS and RFS in osteosarcoma patients. Nevertheless, low immune activity and a high EMT score were associated with significantly poorer OS and RFS in osteosarcoma patients. These findings clarify the predictive of EMT-related gene expression and immune activity for survival and recurrence in osteosarcoma patients.

To further explore the source of EMT-related gene expression, we analyzed the gene expression in microarray datasets from the GEO and human samples that we collected. The expression of EMT-related genes was higher than that of stromal cell genes in the osteosarcoma samples, while the expression of stromal cell genes was higher than that of EMT-related genes in the human stromal samples. The FC of EMT-related genes between tumor and normal (stromal) samples was higher than that in stromal cell genes. Although EMT and stromal activity were highly correlated in osteosarcoma, EMT-related genes were more strongly associated with survival than stromal cell genes. These results suggested that stromal cells may serve as a key source of EMTrelated gene expression in osteosarcoma. Taken together, we show that EMT gene expression and immunity are positively correlated in osteosarcoma yet confer a disparate treatment response and prognostic information. EMT-related gene expression in osteosarcoma may require reinterpretation, given the key contribution of stromal cells to such gene expression. The balance of EMT/stromal versus immunity elements may provide more information about the antitumor immune response than measures of immunity alone.

\section{CONCLUSIONS}

Taken together, the results of our study clarified the relationship among EMT, immune activity, stromal activity and tumor purity in osteosarcoma through an analysis of gene expression 
263

264

265

266

267

268

269

270

271

272

273

274

275

276

277

278

279

280

281

282

283

284

285

286

287

288

289

290

291

292

293

294

295

296

297

298 and evaluation of clinical prognosis. Future research could employ single cell RNA-seq technology to further study these factors in osteosarcoma.

\section{Acknowledgement}

None.

\section{Conflict of interests}

The authors declare that there are no conflicts of interest to report.

\section{REFERENCES}

Biazzo A, and De Paolis M. 2016. Multidisciplinary approach to osteosarcoma. Acta Orthop Belg 82:690-698.

Chang SS. 2019. Re: EMT- and Stroma-Related Gene Expression and Resistance to PD-1 Blockade in Urothelial Cancer. J Urol 202:458. 10.1097/01.JU.0000569196.28580.f2

Gugnoni M, Sancisi V, Gandolfi G, Manzotti G, Ragazzi M, Giordano D, Tamagnini I, Tigano M, Frasoldati A, Piana S, and Ciarrocchi A. 2017. Cadherin-6 promotes EMT and cancer metastasis by restraining autophagy. Oncogene 36:667-677. 10.1038/onc.2016.237

Hanzelmann S, Castelo R, and Guinney J. 2013. GSVA: gene set variation analysis for microarray and RNA-seq data. BMC Bioinformatics 14:7. 10.1186/1471-2105-14-7

Isella C, Terrasi A, Bellomo SE, Petti C, Galatola G, Muratore A, Mellano A, Senetta R, Cassenti A, Sonetto C, Inghirami G, Trusolino L, Fekete Z, De Ridder M, Cassoni P, Storme G, Bertotti A, and Medico E. 2015. Stromal contribution to the colorectal cancer transcriptome. Nat Genet 47:312-319. 10.1038/ng.3224

Isella C, Terrasi A, Bellomo SE, Petti C, Galatola G, Muratore A, Mellano A, Senetta R, Cassenti A, Sonetto C, Inghirami G, Trusolino L, Fekete Z, De Ridder M, Cassoni P, Storme G, Bertotti A, and Medico E. 2016. Corrigendum: Stromal contribution to the colorectal cancer transcriptome. Nat Genet 48:1296. 10.1038/ng1016-1296d

Jiang X, Zhang Z, Song C, Deng H, Yang R, Zhou L, Sun Y, and Zhang Q. 2019. Glaucocalyxin A reverses EMT and TGFbeta1-induced EMT by inhibiting TGF-beta1/Smad2/3 signaling pathway in osteosarcoma. Chem Biol Interact 307:158-166. 10.1016/j.cbi.2019.05.005

Kelly AD, Haibe-Kains B, Janeway KA, Hill KE, Howe E, Goldsmith J, Kurek K, Perez-Atayde AR, Francoeur N, Fan JB, April C, Schneider H, Gebhardt MC, Culhane A, Quackenbush J, and Spentzos D. 2013. MicroRNA paraffinbased studies in osteosarcoma reveal reproducible independent prognostic profiles at 14q32. Genome Med 5:2. 10.1186/gm406

Kotiyal S, and Bhattacharya S. 2014. Breast cancer stem cells, EMT and therapeutic targets. Biochem Biophys Res Commun 453:112-116. 10.1016/j.bbrc.2014.09.069

Kresse SH, Rydbeck H, Skarn M, Namlos HM, Barragan-Polania AH, Cleton-Jansen AM, Serra M, Liestol K, Hogendoorn PC, Hovig E, Myklebost O, and Meza-Zepeda LA. 2012. Integrative analysis reveals relationships of genetic and epigenetic alterations in osteosarcoma. PLoS One 7:e48262. 10.1371/journal.pone.0048262

PeerJ reviewing PDF | (2019:09:41056:1:1:NEW 16 Dec 2019) 
Liu J, Lichtenberg T, Hoadley KA, Poisson LM, Lazar AJ, Cherniack AD, Kovatich AJ, Benz CC, Levine DA, Lee AV, Omberg L, Wolf DM, Shriver CD, Thorsson V, Cancer Genome Atlas Research N, and Hu H. 2018. An Integrated TCGA Pan-Cancer Clinical Data Resource to Drive High-Quality Survival Outcome Analytics. Cell 173:400-416 e411. 10.1016/j.cell.2018.02.052

Lou Y, Diao L, Cuentas ER, Denning WL, Chen L, Fan YH, Byers LA, Wang J, Papadimitrakopoulou VA, Behrens C, Rodriguez JC, Hwu P, Wistuba, II, Heymach JV, and Gibbons DL. 2016. Epithelial-Mesenchymal Transition Is Associated with a Distinct Tumor Microenvironment Including Elevation of Inflammatory Signals and Multiple Immune Checkpoints in Lung Adenocarcinoma. Clin Cancer Res 22:3630-3642. 10.1158/10780432.CCR-15-1434

Lv YF, Dai H, Yan GN, Meng G, Zhang X, and Guo QN. 2016. Downregulation of tumor suppressing STF cDNA 3 promotes epithelial-mesenchymal transition and tumor metastasis of osteosarcoma by the Wnt/GSK3beta/beta-catenin/Snail signaling pathway. Cancer Lett 373:164-173. 10.1016/j.canlet.2016.01.046

Mak MP, Tong P, Diao L, Cardnell RJ, Gibbons DL, William WN, Skoulidis F, Parra ER, Rodriguez-Canales J, Wistuba, II, Heymach JV, Weinstein JN, Coombes KR, Wang J, and Byers LA. 2016. A Patient-Derived, Pan-Cancer EMT Signature Identifies Global Molecular Alterations and Immune Target Enrichment Following Epithelial-toMesenchymal Transition. Clin Cancer Res 22:609-620. 10.1158/1078-0432.CCR-15-0876

Maximov VV, Akkawi R, Khawaled S, Salah Z, Jaber L, Barhoum A, Or O, Galasso M, Kurek KC, Yavin E, and Aqeilan RI. 2019. MiR-16-1-3p and miR-16-2-3p possess strong tumor suppressive and antimetastatic properties in osteosarcoma. Int J Cancer. 10.1002/ijc.32368

Mitschke J, Burk UC, and Reinheckel T. 2019. The role of proteases in epithelial-to-mesenchymal cell transitions in cancer. Cancer Metastasis Rev. 10.1007/s10555-019-09808-2

Paoloni M, Davis S, Lana S, Withrow S, Sangiorgi L, Picci P, Hewitt S, Triche T, Meltzer P, and Khanna C. 2009. Canine tumor cross-species genomics uncovers targets linked to osteosarcoma progression. BMC Genomics 10:625. 10.1186/1471-2164-10-625

Sayles LC, Breese MR, Koehne AL, Leung SG, Lee AG, Liu HY, Spillinger A, Shah AT, Tanasa B, Straessler K, Hazard FK, Spunt SL, Marina N, Kim GE, Cho SJ, Avedian RS, Mohler DG, Kim MO, DuBois SG, Hawkins DS, and SweetCordero EA. 2019. Genome-Informed Targeted Therapy for Osteosarcoma. Cancer Discov 9:46-63. 10.1158/2159-8290.CD-17-1152

Shen S, Huang K, Wu Y, Ma Y, Wang J, Qin F, and Ma J. 2017. A miR-135b-TAZ positive feedback loop promotes epithelial-mesenchymal transition (EMT) and tumorigenesis in osteosarcoma. Cancer Lett 407:32-44. 10.1016/j.canlet.2017.08.005

Singh S, and Chakrabarti R. 2019. Consequences of EMT-Driven Changes in the Immune Microenvironment of Breast Cancer and Therapeutic Response of Cancer Cells. J Clin Med 8. 10.3390/jcm8050642

Subramanian A, Tamayo P, Mootha VK, Mukherjee S, Ebert BL, Gillette MA, Paulovich A, Pomeroy SL, Golub TR, Lander ES, and Mesirov JP. 2005. Gene set enrichment analysis: a knowledge-based approach for interpreting genome-wide expression profiles. Proc Natl Acad Sci U S A 102:15545-15550. 10.1073/pnas.0506580102

Uhlen M, Zhang C, Lee S, Sjostedt E, Fagerberg L, Bidkhori G, Benfeitas R, Arif M, Liu Z, Edfors F, Sanli K, von Feilitzen K, Oksvold P, Lundberg E, Hober S, Nilsson P, Mattsson J, Schwenk JM, Brunnstrom H, Glimelius B, Sjoblom T, Edqvist PH, Djureinovic D, Micke P, Lindskog C, Mardinoglu A, and Ponten F. 2017. A pathology atlas of the human cancer transcriptome. Science 357. 10.1126/science.aan2507

Peer] reviewing PDF | (2019:09:41056:1:1:NEW 16 Dec 2019) 
340

341

342

343

344

345

346

347

348

349

350

Wang L, Saci A, Szabo PM, Chasalow SD, Castillo-Martin M, Domingo-Domenech J, Siefker-Radtke A, Sharma P, Sfakianos JP, Gong Y, Dominguez-Andres A, Oh WK, Mulholland D, Azrilevich A, Hu L, Cordon-Cardo C, Salmon H, Bhardwaj N, Zhu J, and Galsky MD. 2018. EMT- and stroma-related gene expression and resistance to PD-1 blockade in urothelial cancer. Nat Commun 9:3503. 10.1038/s41467-018-05992-x

Yeung KT, and Yang J. 2017. Epithelial-mesenchymal transition in tumor metastasis. Mol Oncol 11:28-39. 10.1002/1878-0261.12017

Yoshihara K, Shahmoradgoli M, Martinez E, Vegesna R, Kim H, Torres-Garcia W, Trevino V, Shen H, Laird PW, Levine DA, Carter SL, Getz G, Stemke-Hale K, Mills GB, and Verhaak RG. 2013. Inferring tumour purity and stromal and immune cell admixture from expression data. Nat Commun 4:2612. 10.1038/ncomms3612 
351

352

353

354

355

356

357

358

359

360

361

362

363

364

365

366

367

368

369

370

371

372

373

374

375

376

377

Figure legends

Fig. 1 EMT-related genes, immune activity and stromal genes in osteosarcoma.

Heatmap showing the expression of EMT-related genes (A and B), immune activity (C and D) and stromal genes (E and F) in osteosarcoma in two independent microarray datasets (GSE39055 on the left and GSE16091 on the right).

Fig. 2 EMT-related gene expression is positively correlated with immunity and stromal activity but negatively correlated with tumor purity.

Plot showing the correlation between EMT and immunity in GSE39055 (A) and GSE16091 (B).

Plot showing the correlation between EMT and stromal activity in GSE39055 (C) and GSE16091

(D). Plot showing the correlation between EMT and tumor purity in GSE39055 (E) and GSE16091

(F). Plot showing the correlation between immunity and tumor purity in GSE39055 (G) and GSE16091 (H). Plot showing the correlation between stromal activity and tumor purity in GSE39055 (I) and GSE16091 (J).

Fig. 3 EMT-related gene expression and immune activity result in disparate clinical outcomes in osteosarcoma patients.

Kaplan-Meier survival curves showing the OS of osteosarcoma patients in GSE39055 divided into two groups by the (A) EMT score, (B) immune score and (C) difference between the immune and EMT score or (D) divided into four groups by both EMT and immune score. Kaplan-Meier survival curves showing the RFS of osteosarcoma patients in GSE39055 divided into two groups by the $(\mathrm{E})$ EMT score, $(\mathrm{F})$ immune score and $(\mathrm{G})$ difference between the immune and EMT score or $(\mathrm{H})$ divided into four groups by both EMT and immune score.

Fig. 4 EMT-related gene expression originates predominantly from stromal cells in osteosarcoma patients.

(A) Bar plot showing the expression of EMT-related and stromal gene signatures in GSE36001 
378 osteosarcoma samples. (B) Scatter plot showing expression grouped by EMT-related and stromal 379 gene signatures in GSE36001 osteosarcoma samples. (C) Bar plot showing the FC in EMT-related 380 and stromal gene signatures in GSE36001. (D) Scatter plot showing the FC in EMT-related and 381 stromal gene signatures in GSE36001. (E and F) Bar plot showing individual EMT-related and 382 stromal genes ranked according to the significance of their association with OS and RFS in 383 GSE39055. Scatter plot showing gene expression grouped by EMT-related and stromal (including 384 both in stromal and EMT) gene signatures in osteosarcoma (G), stromal samples (H) and FC (I) 385 by using RT-qPCR. 


\section{Figure 1}

Expression of EMT-, immune activity and stromal-related genes in osteosarcoma

(A-F) Heatmap showing the expression of EMT (A-B), immune activity (C-D) and stromal-(E-F) related gense in osteosarcoma in two independent microarray datasets (GSE39055 on the left and GSE16091 on the right)

A

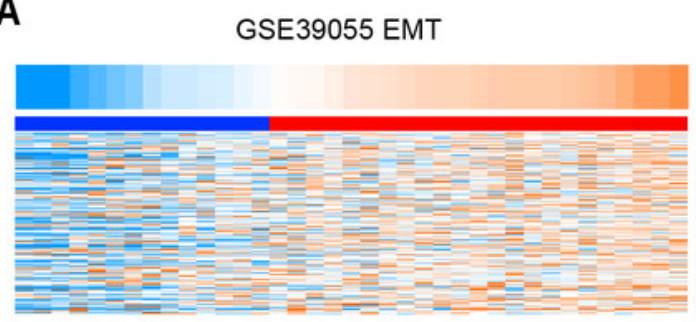

c

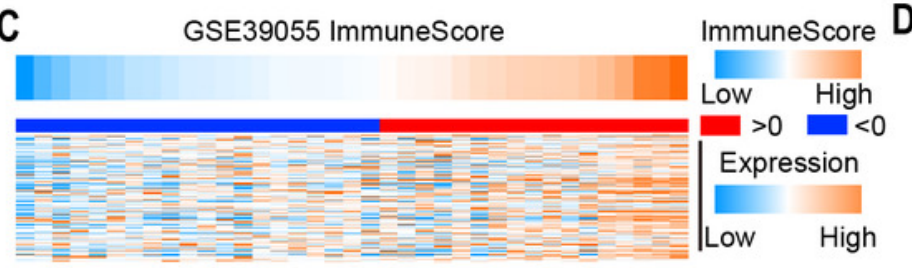

E

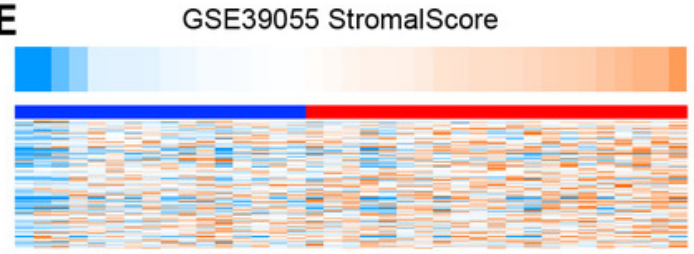

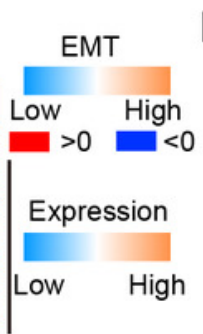

ImmuneScore D

$>0 \quad-<0$

Expression

High
StromalScore $\mathbf{F}$

B
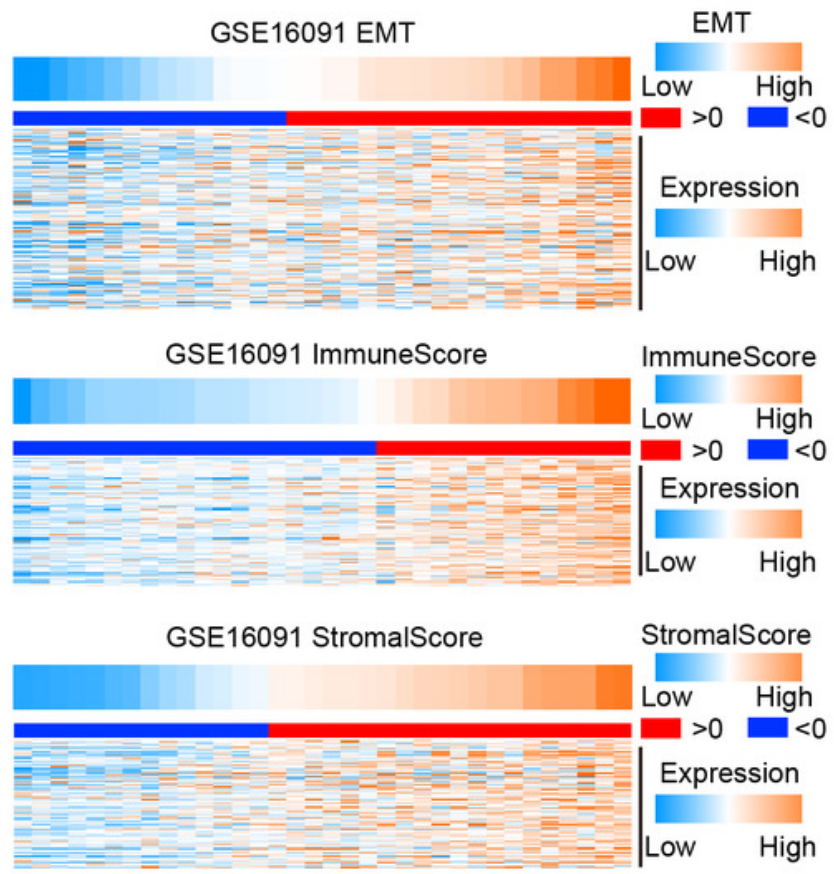
Figure 2

Expression of EMT-related genes is positively correlated with immune and stromal activity but negatively correlated with tumor purity

(A-B) Plot showing the correlation between (A) EMT and immunity and (B) EMT and stromal activity. Left: GSE39055, right: GSE16091. (C-E) Plot showing the correlation between tumor purity and (C) EMT, (D) immunity and (E) stromal activity. Top: GSE39055, bottom: GSE16091
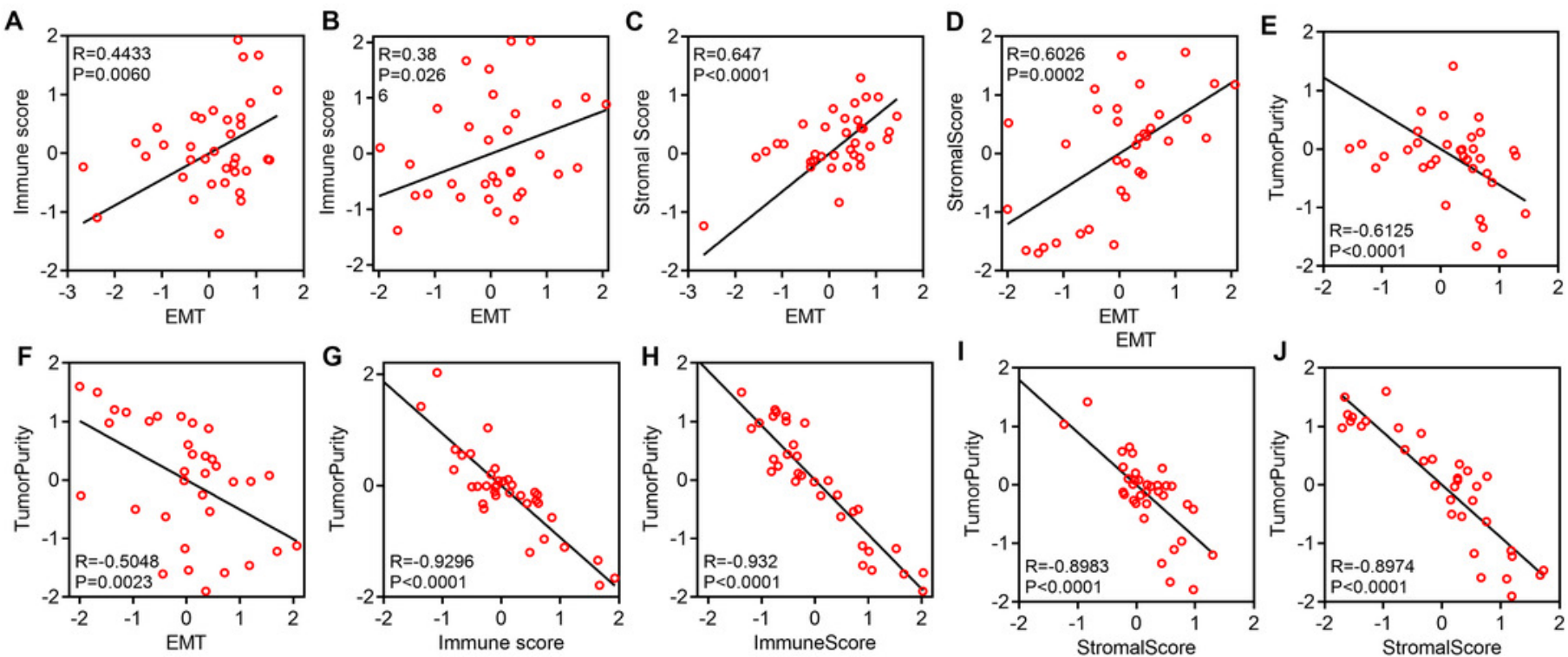


\section{Figure 3}

Expression of EMT-related genes and immune activity result in disparate clinical outcomes in osteosarcoma patients

(A-D) Kaplan-Meier survival curves showing the OS of osteosarcoma patients in GSE39055 divided into two groups by the (A) EMT score, (B) immune score and (C) difference between the immune and EMT score or (D) divided into four groups by both EMT and immune score. (E-H) Similar to A-D, but demonstrate the RFS of osteosarcoma patients in GSE39055 
A

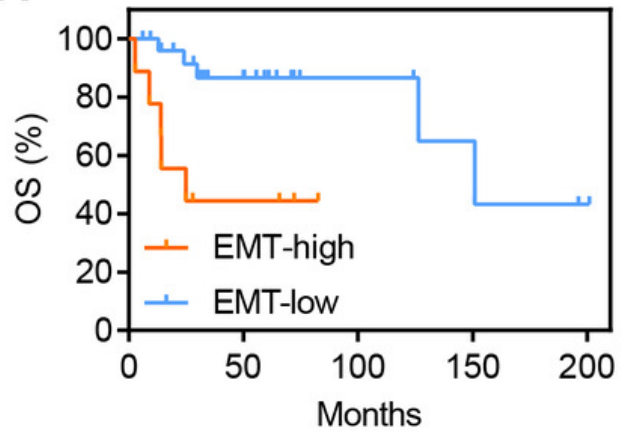

C

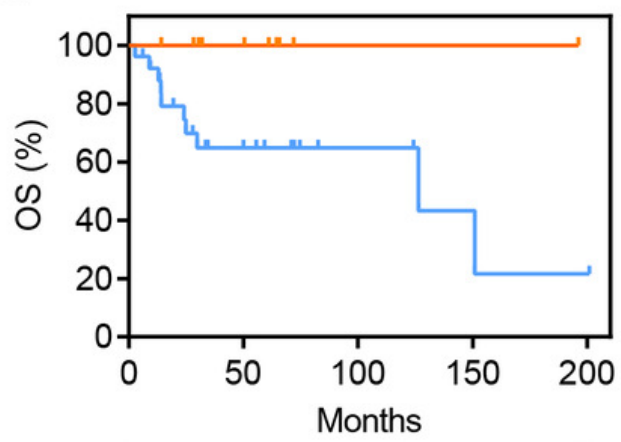

- Difference value of immune to EMT-high

- Difference value of immune to EMT-low

E

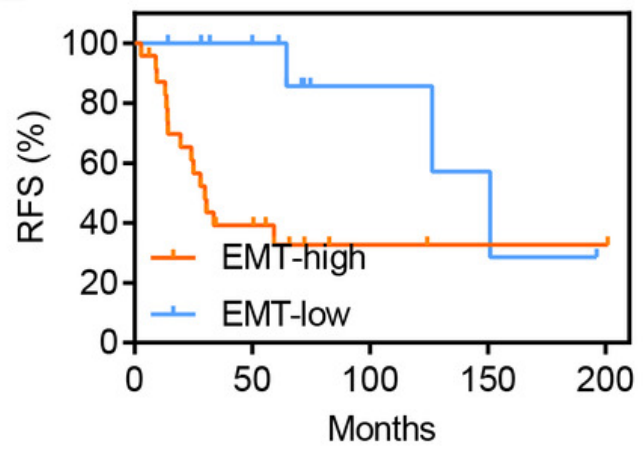

G

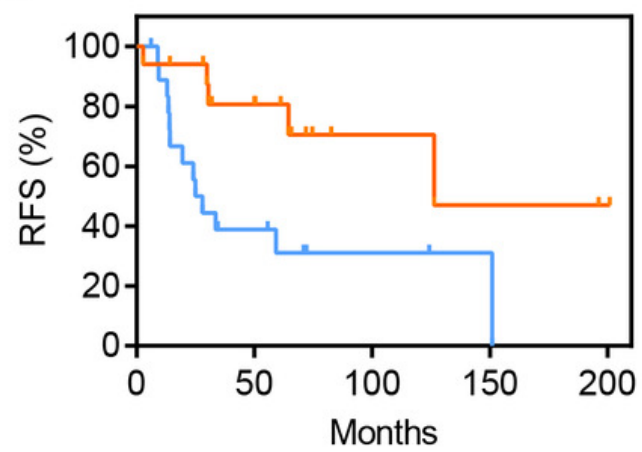

B

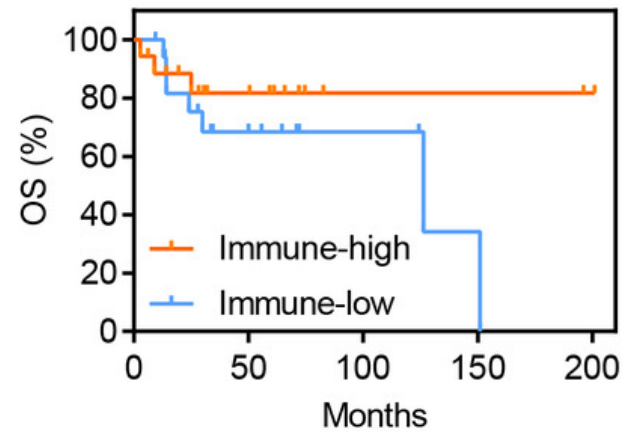

D

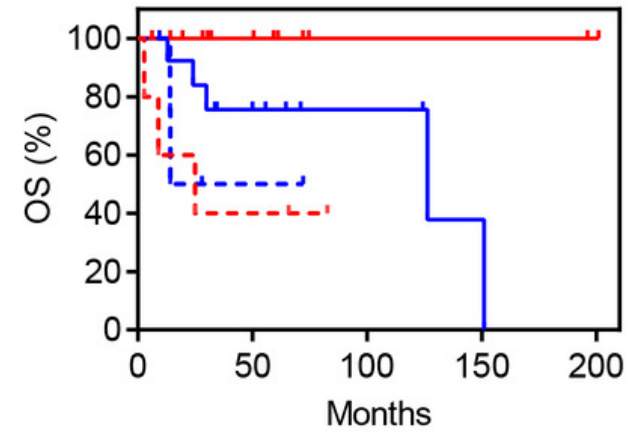

- -. Immune-high EMT-high - Immune-high EMT-low - -. Immune-low EMT-high - Immune-low EMT-low

$\mathbf{F}$

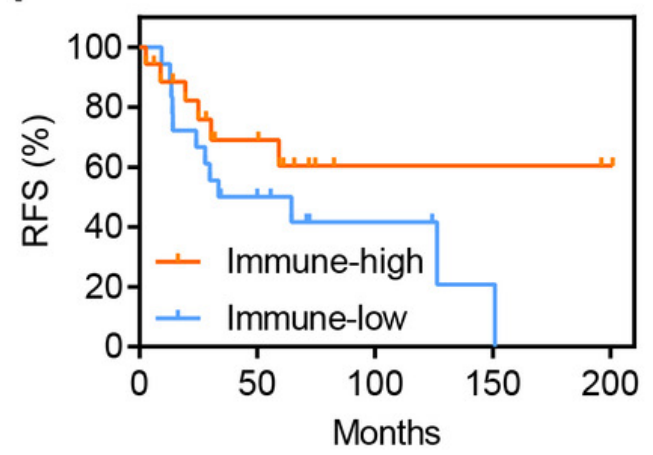

H

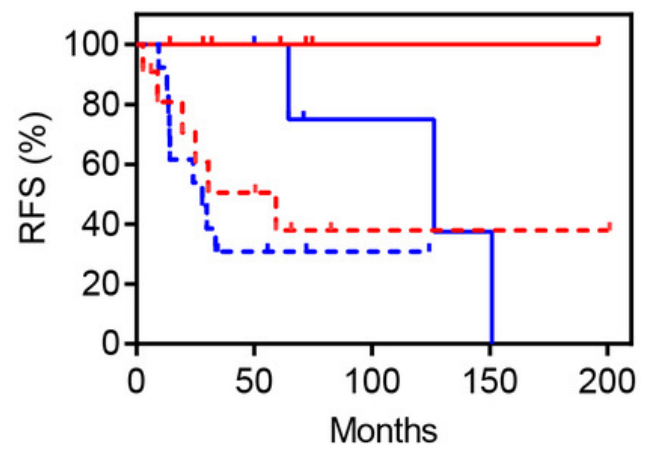

- Difference value of immune to EMT-high - -. Immune-high EMT-high - Immune-high EMT-low

- Difference value of immune to EMT-low - - . Immune-low EMT-high - Immune-low EMT-low 


\section{Figure 4}

EMT-related gene expression originates predominantly from stromal cells in osteosarcoma patients

(A) Bar plot showing the expression of EMT-related and stromal gene signatures in GSE36001 osteosarcoma samples. (B) Scatter plot showing expression grouped by EMT-related and stromal gene signatures in GSE36001 osteosarcoma samples. (C) Bar plot showing the FC in EMT-related and stromal gene signatures in GSE36001. (D) Scatter plot showing the FC in EMT-related and stromal gene signatures in GSE36001. (E-F) Bar plot showing individual EMTrelated and stromal genes ranked according to the significance of their association with OS and RFS in GSE39055. (G-I) Scatter plot showing expression of grouped by EMT-related and stromal (including both in stromal and EMT) gene signatures in osteosarcoma (G), stromal samples (H) and FC (I) by using RT-qPCR 

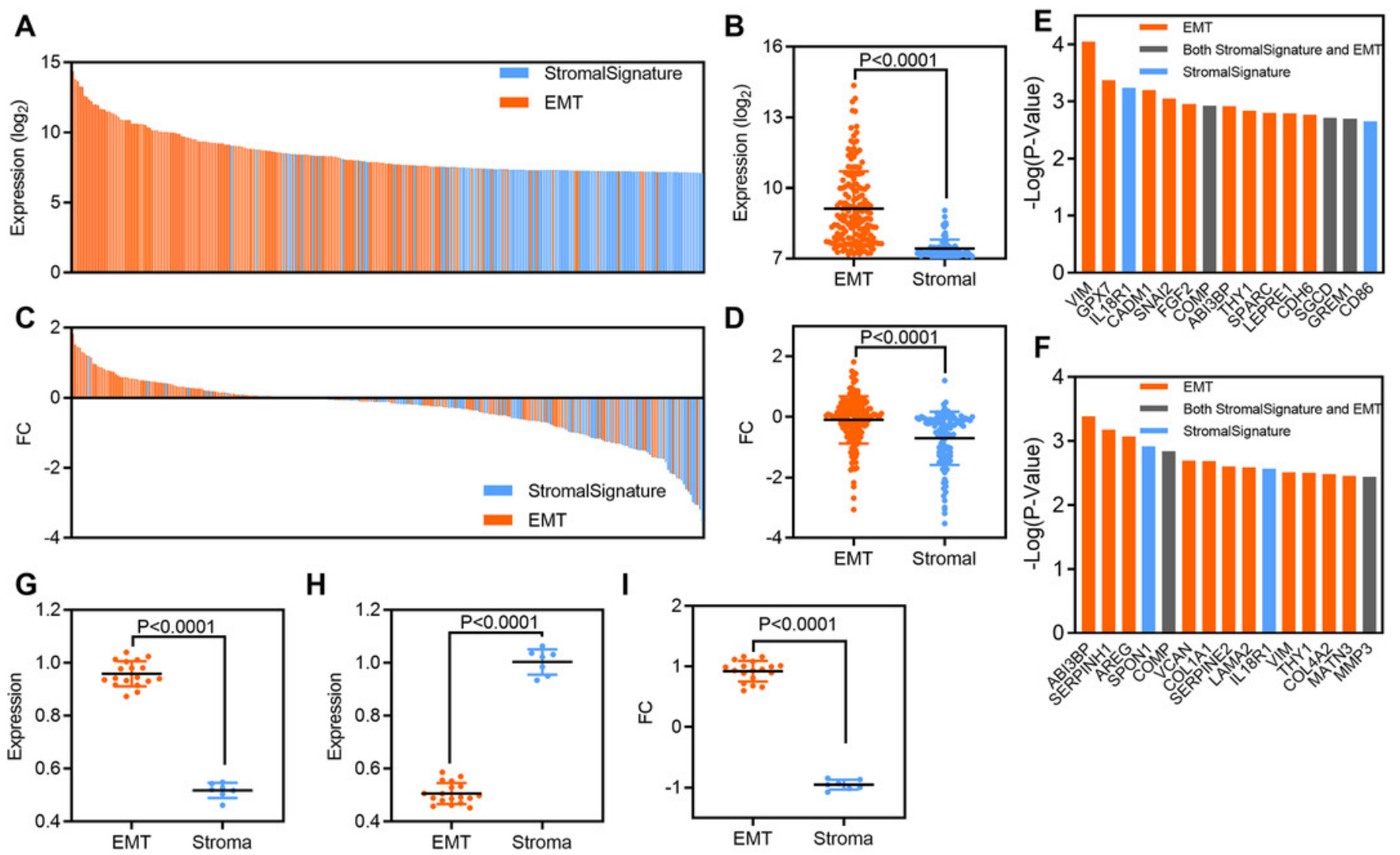